\title{
Graphene-Enabled Improved Supercapacitor Performance of Polyaniline Nanofiber Composites
}

\author{
Ran $\mathrm{Li}^{1}$, Xiaoli Dong ${ }^{1}$, Chengen He ${ }^{1}$, Zixiu Liu ${ }^{1}$, Leping Huang ${ }^{2, *}$, and Yingkui Yang ${ }^{1, *}$ \\ ${ }^{1}$ School of Materials Science and Engineering, Hubei University, Wuhan 430062, China \\ ${ }^{2}$ School of Materials Science and Engineering, Wuhan Textile University, Wuhan 430200, China \\ *E-mail: 1phuang@wtu.edu.cn, ykyang@hubu.edu.cn
}

doi: $10.20964 / 2017.01 .10$

Received: 29 September 2016 / Accepted: 13 November 2016 / Published: 12 December 2016

\begin{abstract}
Graphene/PANI nanofiber composites were synthesized by in-situ chemical oxidative polymerization of aniline in the presence of graphene oxide (GO) followed by chemical reduction of GO. The assynthesized composite was carefully characterized by the combination of the microscopy and spectroscopy techniques. The supercapacitor performance of graphene/PANI nanofiber composite was further investigated. As expected, the synergistic interactions between graphene and PANI enable the as-fabricated electrode with greatly improved electrochemical performance. The specific capacitance of graphene/PANI was calculated to be $952 \mathrm{~F} / \mathrm{g}$ at $1 \mathrm{~A} / \mathrm{g}$, much higher than those of graphene $(236 \mathrm{~F} / \mathrm{g})$ and PANI $(621 \mathrm{~F} / \mathrm{g})$ counterparts. The specific capacitance maintains $645 \mathrm{~F} / \mathrm{g}$ at $10 \mathrm{~A} / \mathrm{g}$ with $68 \%$ retention of original capacitance after a 10-fold increase in the current density. Moreover, the graphene/PANI electrode remains $88 \%$ retention of initial capacitance over 1000 cycles, while PANI only gives $73 \%$ of original capacitance. These data clearly suggest that large capacitance, high rate capability, and good cycling stability can be synchronously achieved by doping PANI nanofibers with graphene sheets.
\end{abstract}

Keywords: Graphene, polyaniline, nanocomposites, supercapacitors

\section{FULL TEXT}

(C) 2017 The Authors. Published by ESG (www.electrochemsci.org). This article is an open access article distributed under the terms and conditions of the Creative Commons Attribution license (http://creativecommons.org/licenses/by/4.0/). 